\title{
CHARACTERIZATION OF COMPOSITE ROD-PINCH-DIODE RADIOGRAPHIC SOURCES AT 5 TO 6 MV ON ASTERIX
}

\author{
D. Mosher ${ }^{+}$, R.J. Allen, R.J. Commisso, S.B. Swanekamp ${ }^{a}$, F.C. Young ${ }^{a}$, and G. Cooperstein \\ Plasma Physics Division, Naval Research Laboratory, Washington, DC 20375, USA
}

\author{
C. Vermare, J. Delvaux, Y. Hordé, E. Merle, R. Nicolas, D. Noré, O. Pierret, Y.R. Rosol, \\ Y. Tailleur, and L. Véron \\ Polygône d'Expérimentation de Moronvilliers, Moronvilliers, France
}

\author{
F. Bayol, A. Garrigues, C. Delbos, and G. Nicot \\ Centre d'Etudes de Gramat, Gramat, France
}

\author{
B. Oliver ${ }^{\text {b }}$, D.V. Rose ${ }^{\text {b }}$, D. Rovang, and J. Maenchen \\ Sandia National Laboratories, Albuquerque, NM 87185, USA
}

\begin{abstract}
Composite-rod-pinch loads on Asterix consisting of hollow aluminum tubes supporting either 1-cm-long, 1mm-diam blunt-end or tapered gold slugs, or 1.5- to 2mm-diam gold spheres are characterized. Composite-slug loads have slightly-lower doses than the 1.6- or $2-\mathrm{mm}$ diam standard rod pinches reported elsewhere and smaller spot sizes, leading to higher measured radiographic figures-of-merit (FOM). The FOM for the gold-sphere loads is substantially-smaller than for the slug loads.
\end{abstract}

\section{INTRODUCTION}

The rod-pinch diode provides an intense, pulsed, smalldiameter, megavolt-bremsstrahlung source for point-projection radiography for which the impedance and spot size are partially decoupled.[1-3] The rod pinch (RP) has been evaluated at 5-6 MV on the Asterix generator [4,5], delivering $100 \mathrm{kA}$ to the diode. The standard RP configuration, consisting of a few-mm-diam tungsten or gold anode rod protruding through, and a few- $\mathrm{cm}$ beyond an annular carbon cathode, is discussed elsewhere, along with the experimental setup and diagnostics.[5] Here, compositeRP loads consisting of hollow aluminum tubes supporting either 1-cm-long, 1-mm-diam gold slugs (blunt-end or tapered) or 1.5- to 2-mm-diam gold spheres are characterized (Fig. 1). Tips protrude 16 to $22 \mathrm{~mm}$ beyond the cathode. Eleven composite-RP shots were conducted during the experiment, a subset of which are discussed here. Composite-rod-pinch electrical coupling, dose, angular dose distribution, and spatial source $x$-ray distributions from on- and off-axis rolled-edgemeasurements are contrasted with those from standardconfiguration loads.[5]
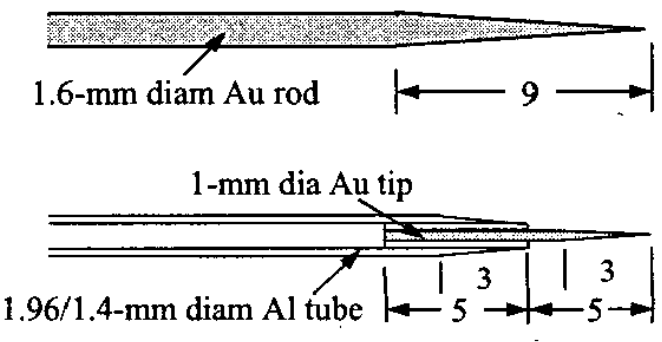

Figure 1. Tapered standard (top) and composite RP loads

Asterix loads require a rod diameter of at least $1.6 \mathrm{~mm}$ under the cathode for good electrical coupling and high dose.[3,5] Smaller diameter in the tip region is required to reduce the spot size and increase the radiographic figure of merit (FOM). The FOM is the dose divided by the square of the LANL-definition source diameter (SD) [2] as viewed from a point on the rod axis $\left(0^{\circ}\right.$ angle). The composite RP, first demonstrated in lower-voltage Asterix experiments [6,7], provides small spot size with a $1-\mathrm{mm}$ diam gold tip and good electrical coupling by transitioning to an aluminum tube under the cathode. The 1.96$\mathrm{mm}-\mathrm{OD}$, 1.4-mm-ID, lower-atomic-number tube minimizes radiation from radii larger than the tip.

Dose is observed [5] and predicted $[8,9]$ to increase with viewing angle. When viewed from $90^{\circ}$, the standard and gold-slug RPs emit over a cm-long region, so that the higher off-axis dose will not be useful for radiography unless a smaller off-axis spot size can be achieved. The gold-sphere-composite loads are designed to address this

\footnotetext{
"Work supported by the Commissariat a l'Energie Atomique/DAM, the Centre d'Etudes de Gramat/DGA, and Sandia National Laboratories/DOE.

+ email: mosher@suzie.nrl.navy.mil

a Titan/Jaycor, McLean, VA 22102.

${ }^{b}$ Mission Research Corporation, Albuquerque, NM 87110.
} 
need. Such a load would be useful for multiple-source radiography geometries that illuminate the target from different directions, and to preserve high radiographic resolution at the edge of extended objects centered on axis.

\section{DISCUSSION AND CONCLUSIONS}

Figures 2 and 3 show the $0^{\circ}$ and $90^{\circ}$ (axial) line spreads (LSs) for standard 1.6-mm-diam Au-rod shots: 7182 with a blunt-end tip, and 7184 with a tip tapered to a point over $10 \mathrm{~mm}$. The $90^{\circ}$ data are acquired from $80^{\circ}$ rolled-edge data corrected to $90^{\circ}$. The $0^{\circ}$ LSs of Fig. 2 have shapes suggested by electron deposition in the rod tip: uniform $x-$ ray emission from a cylinder for 7182 , and from a cone for 7184.[1,5] For the cylinder (a disc viewed on-axis),

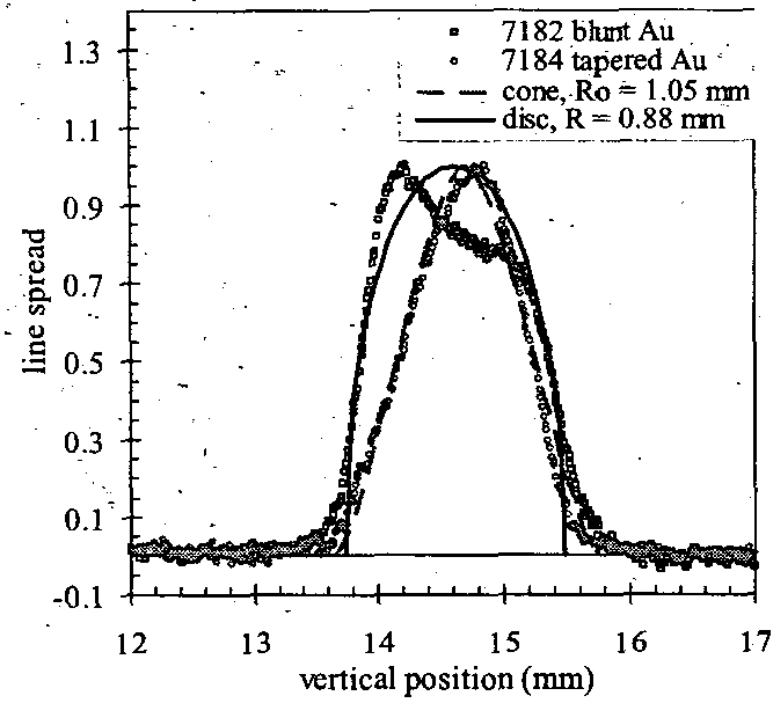

Figure 2. $0^{\circ}$ line spreads for standard 1.6-mm-diam RPs

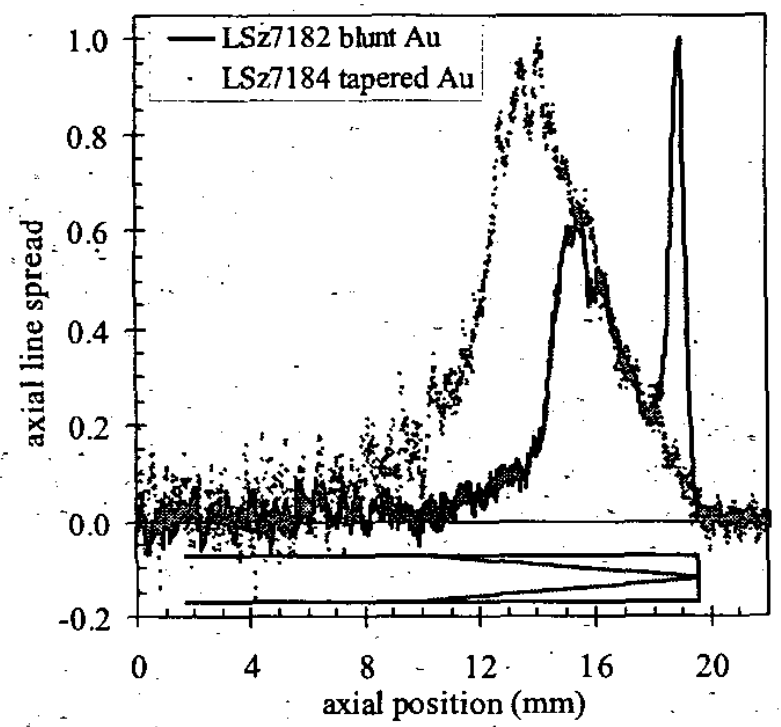

Figure 3. $90^{\circ}$ line spreads for standard 1.6-mm-diam RPs

$$
L S(y)=\sqrt{1-(y / R)^{2}}
$$

where $\mathbf{R}$ is the radius of the cylinder. For the cone,

$$
\begin{aligned}
& L S(y)=\sqrt{1-\left(y / R_{0}\right)^{2}}- \\
& \left(y / R_{0}\right)^{2} \ln \left[\left(R_{0} / y\right)+\sqrt{\left(R_{0} / y\right)^{2}-1}\right]
\end{aligned}
$$

where $R_{0}$ is the radius of the cone base. Fits from Eqns. (1) and (2) are shown in Fig. 2. Values of $R$ and $R_{0}$ in excess of the $0.8-\mathrm{mm}$ rod radius are attributed to hydrodynamic expansion. [9] The LS shape differences lead to a smaller SD for a tapered tip $(1.7 \mathrm{~mm})$ than for a blunt tip $(2.1 \mathrm{~mm})$ with the same rod diameter.

Tip profiles in Fig. 3 are shown below the line spreads on the same axial scale. The spike near the blunt tip is due to electron impact on the front face as predicted in particle-in-cell (PIC) simulations of high-voltage rodpinch flows [8]. Peak emission for the tapered tip is further back because of the small conical emitting volume near the end. It is speculated that the higher mass of the blunt tip allows more complete electron absorption over a shorter axial pinch length, producing a narrower axial LS. For either tip, the axial extent of emission is too large for radiography at large angles to the rod axis. The axial LSs of Fig. 3 are typical for both standard and composite RPs.

The impedance histories for three tapered-gold-tip RPs are shown in Fig. 4. The histories are shifted to align the times of peak x-radiation (arrow). The figure shows the reduced coupling characteristic [4] of the 1-mm-diam standard RP (7175), while the impedances of the 1.6-mmdiam standard (7184) and 1-mm-diam composite (7185) RPs are nearly identical with about 50-Ohm impedance during the radiation pulse.

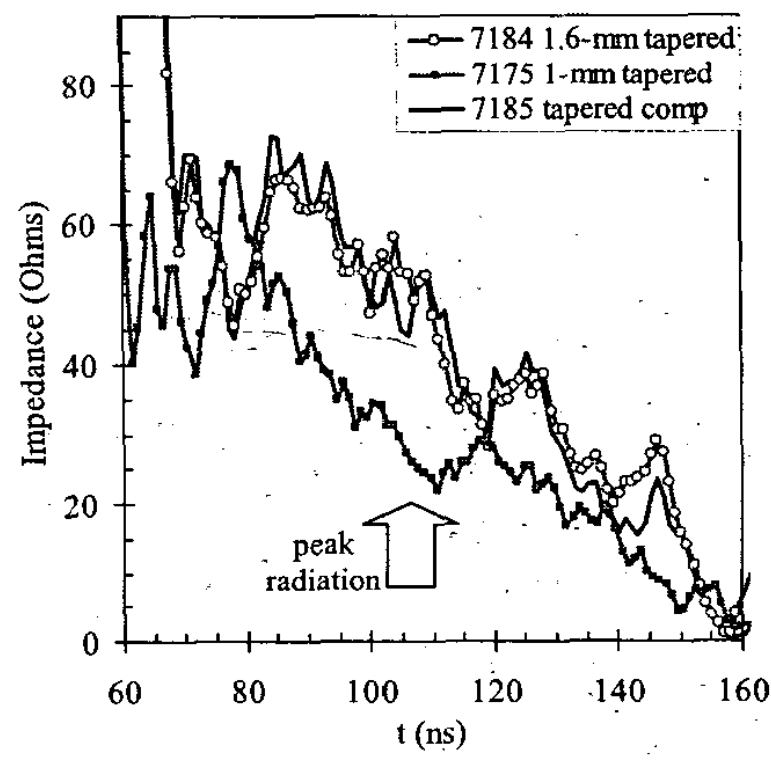

Figure 4. Impedance vs time for three tapered gold RPs 
The reduced impedance of the 1-mm-diam standard RP can be explained in the following way. The impedance scales like $\ln \left(R_{C} / R_{A}\right)$, where the cathode and anode radii vary due to electrode-plasma expansion at speeds $V_{C, A}$.

$$
R_{C}(t)=R_{C O}-V_{C} t \quad ; \quad R_{A}(t)=R_{A 0}+V_{A} t
$$

Typically, $R_{C O} / R_{A O}=11$ provides good coupling in $6-\mathrm{MV}$ Asterix experiments, and all shots discussed here have that value. For small $R_{A O}$, and $V_{A}$ of about $1 \mathrm{~cm} / \mu \mathrm{s}$ [2], $R_{C} / R_{A}$ can change by a large factor during the power pulse. Impedance collapse can also be accelerated by the higher beam heating and faster plasma expansion of a smaller-diameter rod. Increasing $R_{C O} / R_{A O}$ to improve the coupling has limited value because impedance is not well behaved for ratios above $16 .[1,2]$

Figure 5 shows the $0^{\circ}$ line spreads for the geometries of Fig. 4. The 1-mm-diam standard and composite RPs have comparable SDs that are substantially smaller than that of the 1.6-mm-diam standard RP. For 7175 and 7185 , the line spreads show a base width about $0.5-\mathrm{mm}$ larger than the Au tip, suggesting hydrodynamic expansion [10], and for the composite, radiation from the Al tube. The radiographic properties of the three RP configurations are compared in Table 1. Shot 7191 , the same geometry as 7184 , had a $16-\mathrm{mm}$ extension beyond the cathode (7184 had $22 \mathrm{~mm}$ ) and smaller SD. The composite geometry has a dose in air comparable to that of the 1.6-mm-diam standard RP and the spot size of the 1-mm-diam standard, resulting in the largest FOM of all the tested geometries.

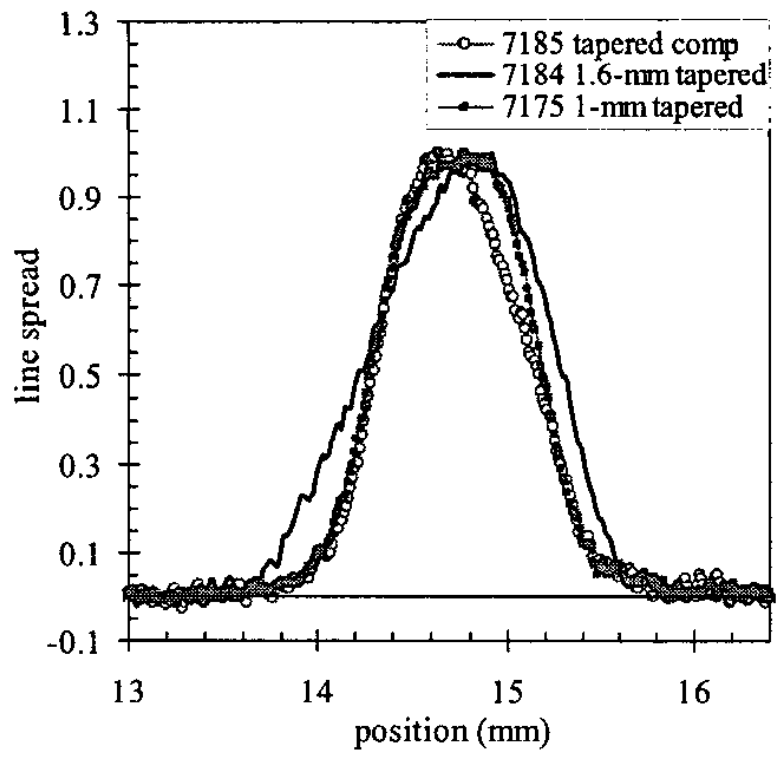

Figure 5. $0^{\circ}$ line spreads for tapered rod pinches

Table 1. Tapered rod pinch properties

\begin{tabular}{|c|c|c|c|}
\hline shot & dose (rad) & SD (mm) & FOM $\left(\mathbf{r a d} / \mathbf{m m}^{2}\right)$ \\
\hline 7185 & 23.7 & 1.32 & 13.6 \\
\hline 7184 & 27.6 & 1.70 & 9.6 \\
\hline 7175 & 13.8 & 1.32 & 7.9 \\
\hline 7191 & 27.4 & 1.55 & 11.4 \\
\hline
\end{tabular}

Figure 6 compares a standard 1.6-mm-diam gold blunttip RP (7182) with a gold blunt-tip composite (7188), and Table 2 compares their radiographic properties. The table also shows the properties of a higher-dose standard 1.6mm-diam tungsten blunt-tip RP (7183). Again, the composite achieves a higher FOM with lower dose because of the reduced SD.

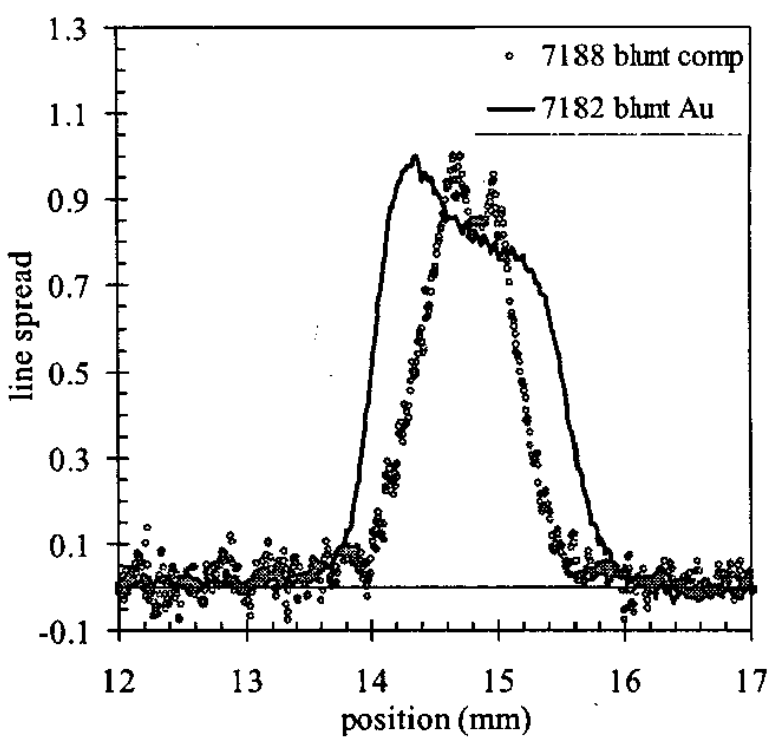

Figure 6. $0^{\circ}$ line spreads for blunt-tip RPs

Table 2. Blunt-tip rod pinch properties

\begin{tabular}{|c|c|c|c|}
\hline shot & dose (rad) & SD (mm) & FOM ( $\left.\mathbf{r a d} / \mathbf{m m}^{2}\right)$ \\
\hline 7182 & 28.5 & 2.10 & 6.5 \\
\hline 7183 & 34.8 & 2.00 & $8.7(\mathrm{~W})$ \\
\hline 7188 & 20.6 & 1.39 & 10.7 \\
\hline
\end{tabular}

The angular variations of dose for the RP geometries discussed above are shown in Fig. 7. The TLD (circles) and PIN-diode (squares) doses are normalized to their values at $20^{\circ}$, where both measurements are made. Both standard [5] and composite RPs show similar scalings, with doses at $90^{\circ}$ a factor 1.6 to 2.1 higher than at $0^{\circ}$. PIC computations [9] of TLD dose vs angle for two rod diameters (dashed lines) [8] agree with the measurements. In order to utilize the higher dose at large angles, the off-axis spot size (Fig. 2) must be greatly reduced.' To this end, 1.5- to 2-mm-diam gold-sphere composites were tested.

Figure 8 compares the $0^{\circ}$ and $90^{\circ}$ line spreads for a gold spheroid with $2-\mathrm{mm}$ vertical diam and $1.5-\mathrm{mm}$ axial diam. Table 3 compares their properties. The $90^{\circ} \mathrm{LS}$ shows the front-surface peaking observed with blunt-tip loads, and its SD is increased by weak emission from the aluminum tube at axial positions less than $15 \mathrm{~mm}$. Emission from the aluminum tube may be due to insufficient gold mass to effectively absorb the electron beam, which then migrates back to the tube. Enhanced beam heating of the tube may lead to increased anode expansion, thereby producing the impedance collapse observed with these loads. Gold-sphere loads behaved similarly in Gamble II 
plasma-filled RP experiments.[11] The $0^{\circ} \mathrm{LS}$ is well represented by that expected for a uniformly-emitting sphere with a radius $R$ slightly-larger than that of the gold spheroid viewed from the rod-pinch axis.

$$
L S(y)=1-(y / R)^{2}
$$

The increased dose and decreased spot size at $90^{\circ}$ produce an FOM about 2.5-times greater than that at $0^{\circ}$.

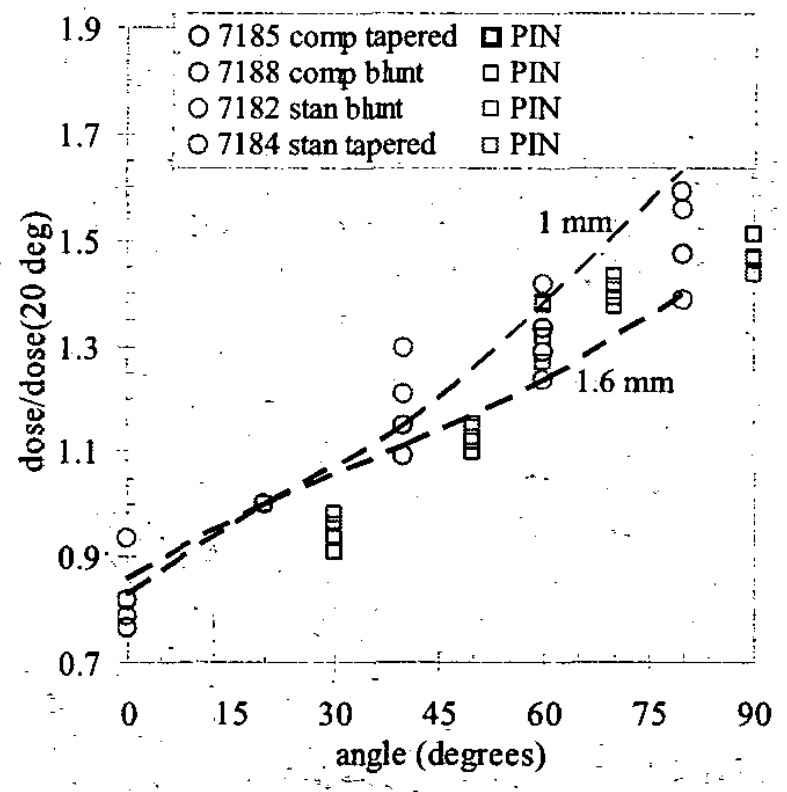

Figure 7. Angular variations of TLD (circles) and PINdiode (squares) dosê. Dashes are predictions from PIC simulations for two rod diamèters.

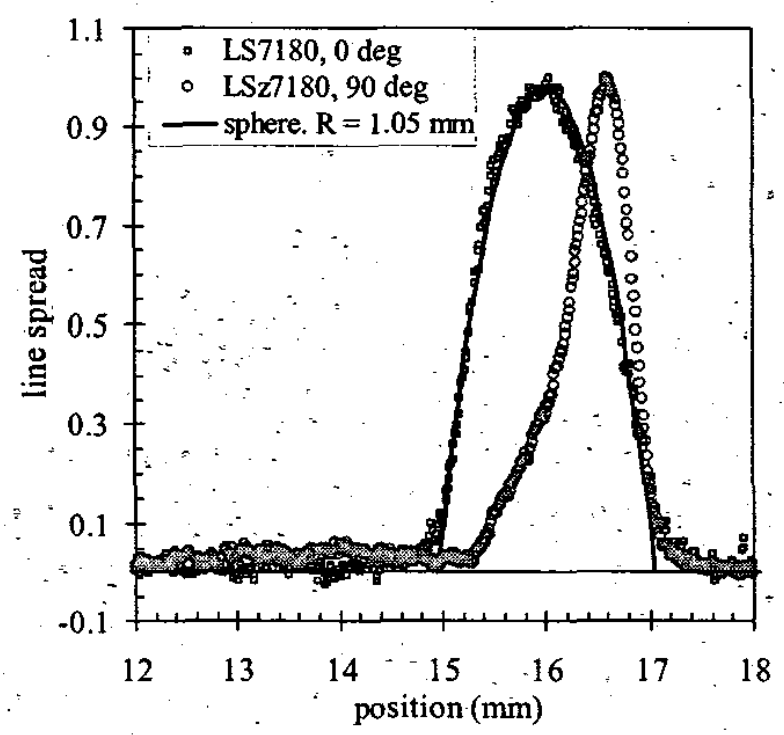

Figure 8. $0^{\circ}$ and $90^{\circ} \mathrm{LS}$ for a gold-shot composite RP
Table 3. Gold-sphere composite properties at $0^{\circ}$ and $90^{\circ}$

\begin{tabular}{|c|c|c|c|}
\hline angle & dose (rad) & SD (mm) & FOM ( $\left.\mathbf{r a d} / \mathbf{m m}^{2}\right)$ \\
\hline 0 deg & 10.2 & 2.16 & 2.2 \\
\hline $90 \mathrm{deg}$ & 16.4 & 1.70 & 5.7 \\
\hline
\end{tabular}

In summary, composite-slug loads have slightly-lower doses than the best-performing standard RPs and smaller spot sizes, leading to higher measured radiographic FOM. For both standard and composite RPs, tapered tips have higher FOMs than blunt tips. The Au-sphere dose and FOM was smaller than for the slug loads, due perhaps to their limited mass and radiating volume. However, limited-mass loads such as the spheres show promise for offaxis radiography, multiple-source radiography geometries that illuminate the target from different directions, and to preserve high radiographic resolution at the edge of extended objects centered on axis. For limited-mass loads, the challenges are: to efficiently couple electron-beam energy to the tip, to minimize deposition in the support tube, and to maintain good impedance behavior. PIC simulations will aid in achieving these objectives.

\section{REFERENCES}

[1] - R.J. Commisso et al., "Experimental evaluation of a megavolt rod-pinch diode as a radiography source," IEEE Trans. Plasma Sci. 30, 338-350(2002).

[2] F.C. Young et al., "Rod Pinch Diode Operation at 2 to $4 \mathrm{MV}$ for High Resolution Pulsed Radiography," Phys. Plasmas 9, 4815-4818(2002), and references therein.

[3] G. Cooperstein, et al., "Theoretical modeling and experimental characterization of a rod-pinch diode," Phys. Plasmas 8, 4618-4636(2001).

[4] R.J. Commisso, et al., these proceedings.

[5] F.C. Young, et al., these proceedings.

[6] F. Bayol, et al., "Evaluation of the rod-pinch diode as a high-resolution source for flash radiography at 2 to 4MV," Proc. 2001 IEEE PPPS Conf. 1, pp. 450-453.

[7] R.J. Commisso, et al., "Characterization of the RodPinch Diode at 2 to $4 \mathrm{MV}$ as a High-Resolution Source for Flash Radiography," AIP Conf. Proc 650, p. 183-6.

[8] S.B. Swanekamp, et al., these proceedings.

[9] D.V. Rose, et al., "Coupled particle-in-cell and Monte Carlo transport modeling of intense radiographic sources," J. Appl. Phys. 91, 3328-3335(2002).

[10]D. Mosher, et al:, "An Electron-Beam-Heating Model for the Gamble II Rod Pinch," AIP Conf. Proc 650, 2002, p. 199-202.

[11]B.V. Weber, et al., "The Plasma-Filled Rod-Pinch Diode: a New Technique to Concentrate MeV Electron Beams to Ultra-High Power- and Energy-Densities," AIP Conf. Proc 650, 2002, p. 191-194. 